This article was published in Journal of Agromedicine, 20(2), 160-166, 2015

http://dx.doi.org/10.1080/1059924x.2015.1010061

\title{
The Epidemiology of Blood-Contaminated Needlestick Injuries Among Veterinarians in Portugal
}

\author{
João R. Mesquita, ${ }^{1,2}$ Sofia I. V. Sousa, ${ }^{3}$ Helena Vala, ${ }^{1,4}$ and Maria S. J. Nascimento ${ }^{2,5}$ \\ ${ }^{1}$ Instituto Politécnico de Viseu, Escola Superior Agrária, Viseu, Portugal \\ ${ }^{2}$ Centro de Investigação em Biodiversidade e Recursos Genéticos, Universidade do Porto, \\ Vairão, Portugal \\ ${ }^{3}$ LEPABE - Laboratory for Process Engineering, Environment, Biotechnology and Energy, \\ Faculty of Engineering, University of Porto, Porto, Portugal \\ ${ }^{4}$ Centro de Estudos em Educação, Tecnologias e Saúde, Instituto Politécnico de Viseu, \\ Viseu, Portugal \\ ${ }^{5}$ Departamento de Ciências Biológicas, Faculdade de Farmácia da Universidade do Porto, Porto, \\ Portugal
}

ABSTRACT. Needlestick injuries (NIs) are considered a substantial occupational health and safety hazard in contemporary health care practice. Unlike human medicine where much effort has been devoted to reduce the incidence of these events, the same aggressive approach has not been used in veterinary medicine. This study investigated the occurrence of blood-contaminated NIs in Portuguese veterinarians. Participants of a veterinary meeting were asked to complete a questionnaire-based sur- vey. Univariate and multivariate logistic regression analyses were performed to produce predicted probabilities for $\mathrm{NI}$ episodes in veterinarians. From the total of 373 enrolled veterinarians, 293 (78.5\%) reported having had at least one $\mathrm{NI}$ during their professional life. Veterinarians working with dogs were more likely to have experienced a NI (adjusted odds ratio [aOR]: 145.74, $P<.001$ ). The high level of NIs observed in these professionals shows that NIs are a potential occupational health problem in Portuguese veterinarians, with the possibility for transmission of haematogenous zoonosis.

\section{INTRODUCTION}

Needlestick injuries (NIs) are today considered a substantial occupational health and safety hazard in contemporary health care practice by acting as a potential route for exposure to, and transmission of, blood-borne infectious diseases among health care workers in both human and veterinary medicine. ${ }^{1-3}$ Several studies have shown the significant burden that NIs currently represent in human health care workers with figures ranging from 100,000 NIs in the United Kingdom (UK) to 800,000 in the United States (US) per year. ${ }^{4,5}$ A more recent report stated that approximately 600,000 health care workers in the US experience percutaneous exposures incidents to blood each year, either by NIs, sharp injuries, or splashes leading to exposure of the skin or mucosa to blood. ${ }^{6}$ However, it is generally accepted that there is underreporting of NIs, ranging from $75 \%$ to $100 \%{ }^{7-9}$

Much effort has been devoted to reduce the incidence of these events in human medicine, but for various reasons, the same aggressive approach has not been used in veterinary medicine. ${ }^{10}$ 
Transmission of blood-borne zoonotic pathogens has generally been considered a minor hazard for veterinarians. Despite the fact that $60 \%$ of known human infectious diseases and more than $75 \%$ of emerging infections are zoonotic in origin, ${ }^{11-13}$ only a few of these animal pathogens are transmitted to humans by the blood-borne route, ${ }^{10}$ which could help explain the lack of importance given to NIs events in veterinary practices. However, some studies have reported blood-borne zoonotic infections in veterinarians after suffering a NI contaminated with zoonotic pathogens with both a high fatality and morbidity potential. ${ }^{14,15}$ Moreover, although medical complications from farm-related NIs seem uncommon, injuries can be debilitating and lead to loss of productivity. ${ }^{16}$ In fact, unintentionally injected adjuvants such as mineral oils are known to cause severe inflammatory responses and generate significant incapacitation. ${ }^{17}$

Furthermore, it is becoming apparent that NIs are relatively common in veterinary practice, but to date, studies on needle handling practices, risk factors, and NIs reporting practices are manifestly lacking. Therefore, this study attempted to fill this knowledge gap by assessing the prevalence of blood-contaminated NIs in veterinary professionals. Behaviors that could be potentially associated with NIs with blood or other bloody body fluids were also studied.

\section{MATERIALS AND METHODS Location and Population}

The study took place in Santa Maria da Feira, Portugal, at the VII Congress of the Montenegro Veterinary Hospital (CMVH) between 12 and 13 February 2011. The CMVH is an annual congress with a target audience of Portuguese veterinarians, and is generally considered the congress with the highest number of participants from the veterinary field in Portugal. The 2011 CMVH had 1,300 participants, its highest attendance ever, which contributed to a substantial enrolment in the study.

\section{Questionnaire}

Participants were fully informed of the aims of the study and methodology and completed an anonymous questionnaire. In order to achieve the highest number of answers to questionnaires, posters were affixed and flyers were introduced in the participants' conference bag. An NI was defined as a puncture or laceration with a needle or other sharp instrument contaminated with blood or other bloody body fluids. Blood and body fluids were defined as blood, breast milk, cerebral spinal fluid, amniotic fluid, pleural fluid, semen, vaginal secretions, synovial fluid, body fluids containing visible blood, saliva associated with dentistry, wound exudates, vomit, diarrhea, and urine. Data obtained via the anonymous questionnaire covered demographic, professional, and personal details. Regarding demographic details the questionnaire included age and sex. The professional details included years in practice, type of practice (dog, cat, exotic, poultry, bovine, swine, equine, sheep), and his- tory of ever suffering a NI with blood from animals and from which animal species was that blood (dog, cat, exotic, poultry, bovine, swine, equine, sheep). Personal details included inter- action with pet animals (dog, cat, exotic, poultry, bovine, swine, poultry, equine, sheep) during childhood ( $<18$ years old). 


\section{Sample Size Calculation}

To determine an adequate sample size to estimate the population prevalence, sample size for a prevalence survey with finite population correction (precision level of 5\%) was calculated. ${ }^{18}$ As no information has been made avail- able on NIs among Portuguese veterinarians, an estimated prevalence of $50 \%$ was assumed, thus yielding the maximum sample size possible. ${ }^{19}$ Official reports from Portugal state that in 2011, 4,202 veterinarians were working in the country. ${ }^{20}$ According to sample size calculation, at least 353 veterinarians would have to be enrolled in this study. To anticipate nonresponse or missing data, possible dropouts, and potential estimation and measurement inaccuracies, the number of participants was oversampled by $5 \%$. Therefore, the number of participants was increased to 373 veterinarians. A total of 373 valid questionnaires were returned from 1,300 eligible participants $(28.7 \%$ response rate). Table 1 presents the characterization of the professionals who answered the questionnaire.

\section{Statistical Analysis}

Univariate and multivariate logistic regression analyses were performed and crude odds ratio (cOR) and adjusted odds ratio (aOR) were calculated to produce predicted probabilities for NI episodes in veterinarians. cOR and aOR were calculated to assess the presence of confounding factors. Variables were considered independent when $\mathrm{cOR}$ and aOR were similar. Likelihoodratio (LR) test was performed to evaluate statistical significance of risk factors with more than 2 levels (years in practice), given its independence from the variables reference level. $P$ values less than .05 were considered statistically significant. All analyses were per- formed using GraphPad Prism version 5.01 soft- ware (GraphPad Software, San Diego, CA) and Epicalc package in the R software (R 2.15.1). ${ }^{21}$

\section{RESULTS}

From the 373 veterinarians, 293 (78.5\%) reported having had at least one NI during their professional work. Of those reporting having had NI, 79\% were female and $74.5 \%$ male veterinarians. Occurrence of NI in veterinarians who experienced animal interaction during childhood (household animal, age $<18$ years old) compared with those who did not interact with animals during childhood was $78.2 \%$ and $70.8 \%$, respectively. Figure 1 shows the distribution of professionals experiencing NIs per years of practice, making it possible to observe that the proportion of veterinarians who experienced NIs increases with years of practice.

The association between the occurrence of NI and the variables - sex, years in practice, animals that have been worked with, and household animal during childhood ( $<18$ years old) - was evaluated by using univariate and multivariate logistic regression models (Table2).

By=univariate-logistic regression analyses, veterinarians with 11 to 20 years of practice, and = with more than 20=years of practice, were positively associated with NIs, (cOR: 10, 95\% confidence interval $[\mathrm{CI}]: 4.21-23.76, P=<.00 \overline{\mathrm{T}}$ and cOR: 4.75, 95\% CI: $1.08-20.93, P=.039$, respectively), when compared with the group with 1 to 10 years of practice. Having worked with dogs was positively associated with the occurrence of NI (cOR: 44.58, 95\% CI: 21.65-91.78, $P<$ .001 ), whereas working with poultry (cOR: $0.32,95 \% \mathrm{CI}: 0.2-0.54, P<.001$ ), bovine (cOR: $0.43,95 \%$ CI: $0.26-0.72, P=.001$ ), and equine (cOR: $0.45,95 \% \mathrm{CI}: 0.27-0.73, P=.001$ ) was negatively associated with episodes of NI. Household sheep during childhood was positively associated with NIs (cOR: 3.93, 95\% CI: $1.37-11.25, P \quad .011$ ), whereas household poultry (cOR: 0.55, 95\% CI: 0.33-0.92, $P$.022) and equine during childhood (cOR: 0.43, 95\% CI: 0.19- 


\section{$0.98, P$}

.045 ) were both found to be negatively associated with NI occurrence.

From the multivariate logistic regression models, several positive associations were found. Veterinarians with 11 to 20 years of practice were associated with NIs (aOR: 16.54, 95\% CI: 3.69-74.26, $P<.001$ ), when compared with the group of 1 to 10 years of practice. Having worked with dogs was found to be a risk factor for NIs (aOR: 145.74, 95\% CI: 40.94-518.78, $P$ $<.001$ ), whereas history of working with poultry was found to be negatively associated with history of NIs (ÆR: 0.3, 95\% CI: 0.1-0.94, $P$.039). Household bovine and sheep during childhood were both found to be positively associated with NI occurrence (aOR: 62.73, 95\% CI: 7.74-508.4 and aOR: 25.55, 95\% CI: 3.75-174.12, respectively, $P<.001$ ). No statistical association was observed with the remaining variables. The presence of confounding effects can be observed due to differences in cOR and aOR for 11 to 20 years in practice, having worked with dogs, and household bovine during childhood.

\section{DISCUSSION}

The present work investigated the expo- sure rate of NIs contaminated with animal blood in Portuguese veterinarians. A cohort of 373 veterinarians was selected, providing an adequate sample size to estimate the NIs prevalence on the veterinarian population. The high incidence of NIs reported in this population (78.5\%) was similar to that described in other studies on veterinary personnel. ${ }^{22-24}$

This study showed that veterinarians with 11 to 20 years of practice were more likely to have experienced NIs than those with 0 to 10 years of practice. This was expected because individuals with more years of profession have a longer history of contact to animals, thus increasing the NI occurrence in their life. Additionally, the increased likelihood of work- related NIs among professionals with longer experience might also be explained by a laxer discipline, making them especially vulnerable in terms of poor occupational health and safety standards, with resulting increased risk for injuries. Multivariate and univariate logistic regression models also showed that veterinarians with 11 to 20 years of practice were more likely to have experienced a NI, when compared with younger veterinarians.

It was interesting to find that working with dogs was a risk factor for NI occurrence, by univariate and multivariate analyses. Dogs are extremely mobile animals that are seldom fully restrained during the course of veterinary care, and always receive individual medication, often parenterally, thus increasing the risk for NI. Other animals are also likely to be improperly constrained and to receive parenteral medication (cats, exotic, bovine, swine, equine, and sheep); however, no statistical association was observed in this study that could have supported this hypothesis. Working with poultry was negatively associated with NI occurrence in veterinarians (by univariate and multivariate analyses), which is not surprising because poultry are easily and continuously constrained for veterinary care and are often medicated through oral administration (via drinking water and feed medication) and not percutaneously, ${ }^{24}$ thus reducing the risk for NIs in veterinarians. A negative association to NI occurrence and history of working with bovine and equine was found when the single-variable analyses were performed. It is tempting to speculate that there is less likelihood for NI events in large animal practices because bovine and equine are less sensitive to venipuncture than dogs and other small animals ${ }^{25}$ and for this reason would most likely have a less exuberant defense reaction upon venipuncture, therefore reducing the 
risk for NIs for veterinarians.

Concerning animal interaction during child- hood, both univariate and multivariate analyses showed that contact with sheep was a risk factor for NIs. Interaction with bovine during childhood was also considered a risk factor but only through multivariate analysis. A possible explanation for these results is that childhood interaction with animals could lead to excess comfort in the manipulation of animals and generate negligent safety behaviors, thus accumulating occupational hazards. Nevertheless, a more controlled study on this topic would be necessary to make more definite conclusions.

Some studies have reported blood-borne zoonotic infections acquired by veterinarians through NIs. An accidental infection with Herpesvirus simiae was described in a 26-year- old veterinary technician after a NI contaminated with blood from a rhesus monkey. ${ }^{14}$ A blastomycosis accidental infection in a veterinarian was reported to be due to a fine-needle aspiration during the course of a necropsy on a dog. ${ }^{26}$ In Australia, a recent NI of a veterinarian while euthanizing a horse positive for Hendra virus, an emerging paramyxovirus with a high case fatality rate, highlighted the greater danger that veterinarians now face. ${ }^{27} \mathrm{~A}$ case of meningoencephalitis due to West Nile virus (WNV) in a 29-year-old immunocompetent female scientist at the National Institute for Communicable Disease, South Africa, was reported following a NI with neuroinvasive lineage II WNV strain. ${ }^{15}$ More recently, Bartonella spp. infections in veterinarians have also been implicated with accidental NIs. ${ }^{28,29}$

Reports of communicable diseases acquired by NIs in veterinarians are rare, and probably for this reason blood-borne transmission of zoonotic pathogens have generally been considered a minor hazard for veterinarians. Nevertheless, underreporting might be present, and if individual risk analyses underestimate the real risk, such underreporting might embody a missed opportunity for postexposure prophylaxis. Therefore, great attention should be paid to any NI episode given the potential biohazard of blood-borne zoonotic infections. Additionally, other medical complications such as severe inflammation can occur with accidental injection of mineral oils. ${ }^{16,17}$ Further work should also approach these complications.

\section{CONCLUSION}

To our knowledge, this is the first study investigating the exposure to NIs among Portuguese veterinarians, as well as determining risk factors for this work-related exposure. The high level of NIs observed in these professionals shows that NIs are a potential occupational health problem in Portuguese veterinarians, and continuing education in the prevention of percutaneous injuries would be beneficial. Guidelines and strategies to reduce NIs in veterinary practice should be promoted, and the negligent behaviors concerning these injuries should be replaced with a proactive attitude towards injury prevention. Additionally, health and safety refresher courses could have a positive effect on safety culture change and gradually reduce work-related injuries in the future.

\section{Ethical Standards}

Informed consent was obtained from the participants of the study. Survey procedures were conducted in accordance with the recommendations outlined in the Declaration of Helsinki and were approved by the ethics board at the University of Porto (approval number: 18/CEUP/2011). 


\section{FUNDING}

This work was supported by the award number Ovislab ICT-2013-05-004-5314 ID-64757 from QREN/FEDER and the award number SFRH/BPD/64686/2009 from Fundação para a Ciência e Tecnologia (FCT).

\section{REFERENCES}

1. Lee JM, Botteman MF, Xanthakos N, Nicklasson L. Needlestick injuries in the United States. Epidemiologic, economic and quality of life issues. AAOHN J. 2005;53:117-133.

2. Falagas ME, Karydis I, Kostogiannou I. Percutaneous exposure incidents of the health care personnel in a newly founded tertiary hospital. a prospective study. PLoS ONE.2007;2:e194.

3. Weiss ES, Cornwell EE, Wang T, et al. Human immunodeficiency virus and hepatitis testing and preva- lence among surgical patients in an urban university hos- pital. Am J Surg. 2007;193:55-60.

4. National Institute for Occupational Safety and Health (NIOSH). NIOSH Alert. Preventing Needlestick Injuries in Health Care Settings. Cincinnati, OH: National Institute for Occupational Safety and Health; 1999. NIOSH Publication No. 2000-108.

5. van Wijk PT, Schneeberger PM, Heimeriks K, et al. Occupational blood exposure accidents in The Netherlands. Eur J Public Health.2010;20:281-287.

6. Foley M. Update on needlestick and sharps injuries: the Needle Stick Safety and Prevention Act of 2000. Am J Nurs. 2004;104:96.

7. Haiduven D, Simpkins S, Phillips E, Stevens D. A survey of percutaneous/mucocutaneous injury reporting in a public teaching hospital. $J$ Hosp Infect. 1999;41:151-154.

8. Elder A, Paterson C. Sharps injuries in UK health care: a review of injury rates, viral transmission and potential efficacy of safety devices. Occup Med (Lond). 2006;56:566-574.

9. Wicker S, Jung J, Allwinn R, Gottschalk R, Rabenau HF. Prevalence of needlestick injuries among health care workers in a German university hospital. Int Arch Occup Environ Health. 2008;81:347-354.

10. Weese JS, Jack DC. Needlestick injuries in veteri- nary practice. Can Vet J. 2008;49:780-784.

11. Chomel BB. New emerging zoonoses: a challenge and an opportunity for the veterinary profession. Comp Immunol Microbiol Infect Dis. 1998;21:1-14.

12. Taylor LH, Latham SM, Woolhouse MEJ. Risk fac- tors for human disease emergence. Philos Trans $R$ Soc Lond B Biol Sci. 2001;356:983-989.

13. King DA, Peckham C, Waage JK, Brownlie J, Woolhouse ME. Epidemiology. Infectious diseases: prepar- ing for the future. Science. 2006;313:1392-1393.

14. Artenstein AW, Hicks CB, Goodwin BS, Hilliard JK. Human infection with B virus following a needlestick injury. Rev Infect Dis. 1991;13:288-291.

15. Venter M, Swanepoel R. West Nile virus lineage 2 as a cause of zoonotic neurological disease in humans and horses in southern Africa. Vector Borne Zoonotic Dis. 2010;10:659-664.

16. Jennissen C, Wallace J, Donham K, Rendell D, Brumby S. Unintentional needlestick injuries in livestock production: a case series and review. J Agromedicine. 2011;16:58-71.

17.Donham K, Thelin A. Agricultural Medicine: Occupational and Environmental Health for Rural Health Practitioners. Ames, IA: Blackwell Press; 2006: 341-345.

18. Daniel WW. Biostatistics: A Foundation for Analysis in the Health Sciences. New York: John Wiley \& Sons; 1999.

19. Macfarlane SB. Conducting a descriptive survey: 2. Choosing a sampling strategy. Trop Doct. 1997;27:14-21.

20. Ordem dos Médicos Veterinários. Ordem dos Médicos Veterinários-Estatísticas. Available at: http:// www.omv.pt/ordem/estatisticas. Published 2012. Accessed June 10, 2012.

21.R Development Core Team. $R: A$ Language and Environment for Statistical Computing [computer software]. Vienna, Austria: R Foundation for Statistical Computing 3-900051-07-0; 2012. Available at: http:// www.Rproject.org/. Accessed June 4, 2012.

22. Wilkins JR, Bowman ME. Needlestick injuries among female veterinarians: frequency, syringe contents and sideeffects. Occup Med.1997;47:451-457.

23.Leggat PA, Smith DR, Speare R. Exposure rate of needlestick and sharps injuries among Australian veterinarians. J Occup Med Toxicol.2009;4:25-31.

24. Vermeulena B, De Backerb P, Remona JP. Drug administration to poultry. Adv Drug Deliv Rev. 2002;54:795803. 
25. Raekallio M, Heinonen KM, Kuussaari J, Vainio O. Pain alleviation in animals: attitudes and practices of Finnish veterinarians. Vet J. 2003;165:131-135.

26. Ramsey D. Blastomycosis in a veterinarian. J Am Vet Med Assoc. 1994;205:968.

27.Perkins N. Independent Review of Hendra Virus Cases at Redlands and Proserpine in July and August 2008. Toowoomba, Australia: Ausvet Animal Health Services; 2008.

28. Oliveira AM, Maggi RG, Woods CW, Breitschwerdt EB. Suspected needle stick transmis- sion of Bartonella vinsonii subspecies berkhoffii to a veterinarian. J Vet Intern Med.2010;24:1229-1232.

29. Lin JW, Chen CM, Chang CC. Unknown fever and back pain caused by Bartonella henselae in a vet- erinarian after a needle puncture: a case report and literature review. Vector Borne Zoonotic Dis. 2011;11: 589-591.

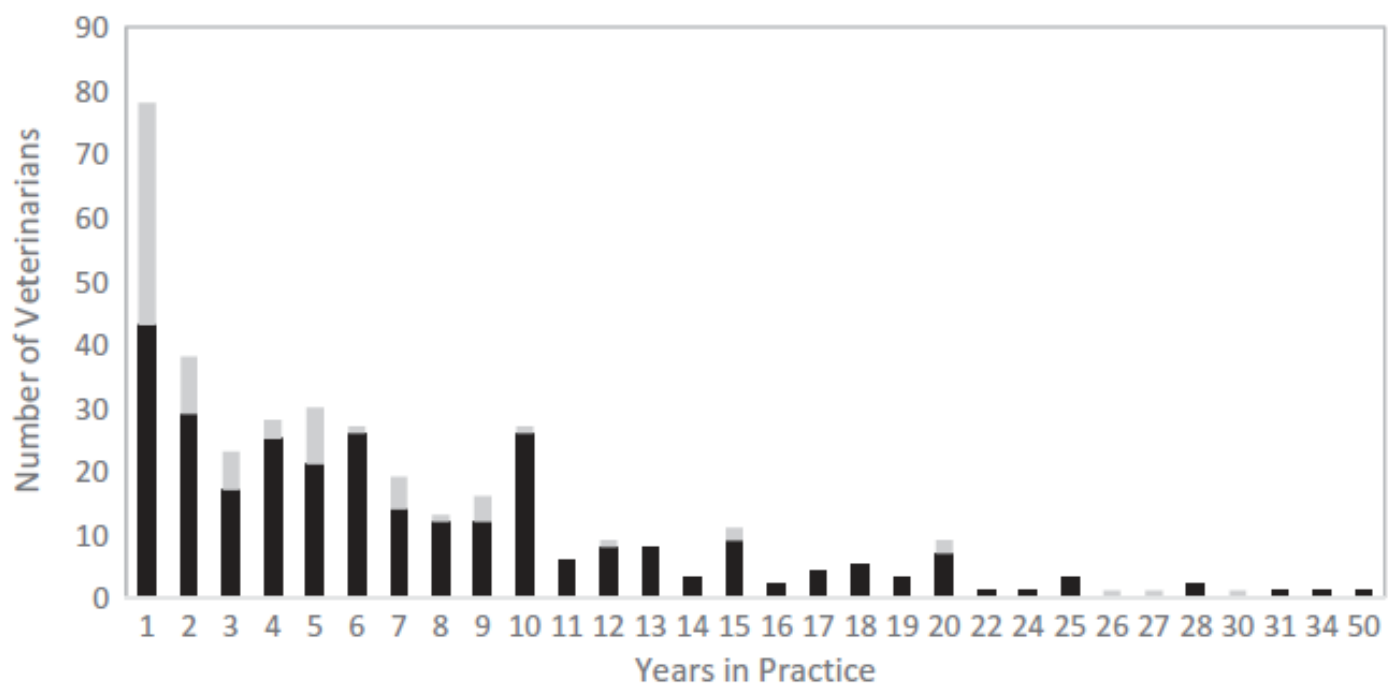

Professionals that experienced NIs

Professionals that did not experience NIs

FIGURE 1. Distribution of professionals experiencing needlestick injuries (NIs) per years of practice. 
TABLE 1. Descriptive Analysis of the Veterinarians Group Under Study $(N=373)$

\begin{tabular}{lrc}
\hline Characteristic & $n / \%$ & $95 \% \mathrm{Cl}$ \\
\hline Sex & & \\
Female & $263 / 70.5 \%$ & $65.9-75.1$ \\
Male & $110 / 29.5 \%$ & $24.9-34.1$ \\
Years in practice & & \\
$1-10$ & $300 / 80.4 \%$ & $76.1-84.2$ \\
$11-20$ & $61 / 16.3 \%$ & $12.4-19.8$ \\
$>20$ & $12 / 3.2 \%$ & $1.8-5.7$ \\
Animals that have been & & \\
worked with & & \\
Dog & & \\
Cat & $264 / 70.7 \%$ & $66.1-75.4$ \\
Exotic & $360 / 96.5 \%$ & $94.9-98.2$ \\
Poultry & $192 / 51.4 \%$ & $47.0-56.0$ \\
Bovine & $134 / 35.9 \%$ & $31.0-40.8$ \\
Swine & $141 / 37.8 \%$ & $33.5-42.1$ \\
Equine & $103 / 27.6 \%$ & $23.6-31.6$ \\
Sheep & $109 / 29.2 \%$ & $25.1-33.3$ \\
Household animal during & $55 / 14.7 \%$ & $11.6-17.9$ \\
childhood $(<18$ years old) & & \\
No & & \\
Yes & $24 / 6.4 \%$ & $4.2-8.6$ \\
$\quad$ Dog & $349 / 93.5 \%$ & $91.4-95.8$ \\
Cat & $315 / 84.4$ & $81.2-87.7$ \\
Exotic & $218 / 58.4 \%$ & $54.0-62.9$ \\
Poultry & $63 / 16.8 \%$ & $13.5-20.2$ \\
$\quad$ Bovine & $115 / 30.8 \%$ & $26.1-35.5$ \\
$\quad$ Ewine & $40 / 10.7 \%$ & $7.9-13.5$ \\
$\quad$ Sheep & $40 / 10.7 \%$ & $7.9-13.5$ \\
\hline & $52 / 13.9 \%$ & $10.4-17.4$ \\
\hline
\end{tabular}

Note. $\mathrm{NI}=$ needlestick injury; $\mathrm{Cl}=$ confidence interval; $n=$ number of individuals 
TABLE 2. Univariate and Multivariate Analyses of Risk Factors for Needlestick Injuries Among Portuguese Veterinarians

\begin{tabular}{|c|c|c|c|c|c|}
\hline \multirow{2}{*}{ Variable } & \multicolumn{2}{|c|}{ Univariate analysis } & \multicolumn{3}{|c|}{ Multivariate analysis } \\
\hline & $\operatorname{cOR}(95 \% \mathrm{Cl})$ & $P$ & $\operatorname{aOR}(95 \% \mathrm{Cl})$ & $P$ & $P(\mathrm{LR})$ \\
\hline Sex & & & & & .255 \\
\hline Male & Ref & & Ref & & \\
\hline Female & $0.78(0.46-1.3)$ & .346 & $0.56(0.21-1.51)$ & .255 & \\
\hline Years in practice & & & & & $<.001$ \\
\hline $1-10$ & Ref & & Ref & & \\
\hline $11-20$ & $10(4.21-23.76)$ & $<.001$ & $16.54(3.69-74.26)$ & $<.001$ & \\
\hline$>20$ & $4.75(1.08-20.93)$ & .039 & $4.87(0.46-51.13)$ & .187 & \\
\hline \multicolumn{6}{|c|}{$\begin{array}{l}\text { Animals that have been worked } \\
\text { with }\end{array}$} \\
\hline Dog & 44.58 (21.65-91.78) & $<.001$ & $145.74(40.94-518.78)$ & $<.001$ & $<.001$ \\
\hline Cat & $2.25(0.72-7.08)$ & .165 & $0.89(0.06-12.33)$ & .932 & .932 \\
\hline Exotic & $1.11(0.68-1.8)$ & .687 & $2.18(0.79-6.02)$ & .132 & .128 \\
\hline Poultry & $0.32(0.2-0.54)$ & $<.001$ & $0.3(0.1-0.94)$ & .039 & .034 \\
\hline Bovine & $0.45(0.27-0.73)$ & .001 & $0.34(0.08-1.38)$ & .131 & .13 \\
\hline Swine & $0.6(0.35-1)$ & .052 & $0.71(0.18-2.78)$ & .623 & .621 \\
\hline Equine & $0.43(0.26-0.72)$ & .001 & $2.32(0.68-7.86)$ & .177 & .172 \\
\hline Sheep & $0.79(0.41-1.54)$ & .491 & $1.75(0.49-6.22)$ & .384 & .377 \\
\hline \multicolumn{6}{|c|}{$\begin{array}{l}\text { Household animal during } \\
\text { childhood ( }<18 \text { years old })\end{array}$} \\
\hline No & Ref & & & & \\
\hline Yes & $1.47(0.59-3.68)$ & .407 & $5.04(0.45-56.63)$ & .19 & .187 \\
\hline Dog & $1.4(0.74-2.65)$ & .295 & $1.07(0.24-4.81)$ & .927 & .927 \\
\hline Cat & $0.96(0.59-1.58)$ & .883 & $0.71(0.26-1.97)$ & .514 & .512 \\
\hline Exotic & $0.88(0.46-1.67)$ & .697 & $0.49(0.13-1.85)$ & .292 & .292 \\
\hline Poultry & $0.55(0.33-0.92)$ & .022 & $0.6(0.2-1.8)$ & .361 & .359 \\
\hline Bovine & $1.65(0.67-4.1)$ & .276 & $62.73(7.74-508.4)$ & $<.001$ & $<.001$ \\
\hline Swine & $0.95(0.43-2.1)$ & .903 & $0.34(0.06-2)$ & .231 & .232 \\
\hline Equine & $0.43(0.19-0.98)$ & .045 & $0.32(0.06-1.78)$ & .195 & .191 \\
\hline Sheep & $3.93(1.37-11.25)$ & .011 & $25.55(3.75-174.12)$ & $<.001$ & $<.001$ \\
\hline
\end{tabular}

Note. $\mathrm{cOR}=$ crude odds ratio; $\mathrm{aOR}=$ adjusted odds ratio; $\mathrm{Ref}=$ variables reference level; $\mathrm{Cl}=$ confidence interval. 